\title{
Lateral air cavities for microfluidic pumping with the use of acoustic energy
}

\author{
Armando R. Tovar • Maulik V. Patel • \\ Abraham P. Lee
}

Received: 16 October 2010/ Accepted: 15 December 2010/Published online: 5 January 2011

(C) The Author(s) 2011. This article is published with open access at Springerlink.com

\begin{abstract}
An acoustically activated micropump is fabricated and demonstrated using a single step lithography process and an off-chip acoustic energy source. Using angled lateral cavities with trapped air bubbles, acoustic energy is used to oscillate the liquid-air interface to create a fluidic driving force. The angled lateral cavity design allows for fluid rectification from the first-order pulsatile flow of the oscillating bubbles. The fluid rectification is achieved through the asymmetrical flow produced by the oscillating interface generating fluid flow away from the lateral cavity interface. Simulation and experimental results are used to develop a pumping mechanism that is capable of driving fluid at pressures of $350 \mathrm{~Pa}$. This pumping system is then integrated into a stand-alone battery operated system to drive fluid from one chip to another.
\end{abstract}

Keywords Micropumps · Acoustic - Simulations · Microstreaming

\section{Introduction}

Over the past 20 years, great strides have been made in developing a portable micro total analysis system using microfluidic technology. However, one major challenge

Electronic supplementary material The online version of this article (doi:10.1007/s10404-010-0758-1) contains supplementary material, which is available to authorized users.

A. R. Tovar $(\bowtie) \cdot$ M. V. Patel · A. P. Lee

Department of Biomedical Engineering, University of California

Irvine, 3406 Engineering Hall, Irvine, CA 92617, USA

e-mail: tovara@uci.edu

URL: http://biomint.eng.uci.edu has been developing an adequate cross-over mechanism between the macro and micro environments that exists when trying to introduce samples of $\mu \mathrm{l}$ and $\mathrm{ml}$ volumes into devices that manipulate one to three orders smaller. The main component that bridges these environments is the fluidic driving mechanism or pump. Since the advent of microfluidics, there have been great advances in this field (Laser and Santiago 2004) that have ranged from using pneumatic and peristaltic pumping (Unger et al. 2000; Chiu and Liu 2009), electrokinetic pumping (Zeng et al. 2001), magnetohydrodynamic pumping (Lemoff and Lee 2000), to using diodes embedded in channel walls (Chang et al. 2008), and using electrolysis to generate bubbles to pump blood (Chiu and Liu 2009). One major limitation that persists in the pumping mechanisms developed is they often require complex fabrication techniques, severely limiting their applications in portable, low-cost systems. In the case of portable diagnostic systems, a pumping mechanism that is simple to operate, fabricate, and compatible with biological samples is essential. Though many pumping technologies exist, an often used fluid transport mechanism in these systems is the use of capillary forces (Gervais and Delamarche 2009; Zimmermann et al. 2009) to achieve fluid delivery. This form of fluid transport is advantageous in that it (1) requires little to no additional fabrication steps, (2) is simple to operate, and (3) requires no additional form of energy applied to the system for operation. The main drawback to this form of fluid transport is the limited control of the fluid flow. Since fluid flow is achieved through surface energy present in the channel materials, it is difficult to start and stop flow on demand, adjust flow rate, and sequentially drive different reservoirs of fluid into a microfluidic system. To address these issues, the coupling of air bubbles with acoustic energy is investigated as a fluidic driving mechanism. 
Traditionally, in the field of microfluidics, the presence of air bubbles or air pockets in microchannels is considered as an unwanted byproduct. However, recently there has been a growing trend in harnessing this byproduct for a variety of novel applications (Rife et al. 2000; Tsai and Lin 2002; Marmottant and Hilgenfeldt 2003, 2004; Dijkink et al. 2006; Marmottant et al. 2006; Hettiarachchi et al. 2007; Kao et al. 2007; Tho et al. 2007; Xu and Attinger 2007; Chung and Cho 2008; Ahmed et al. 2009a, b; Chung and Cho 2009; Tovar and Lee 2009). The first practical use of trapped air bubbles in a microfluidic device utilized acoustic energy to rapidly mix two fluids within a chamber (Liu et al. 2002) for the increase of DNA hybridization (Liu et al. 2003a, b). The combination of acoustic energy and trapped air bubbles within these microsystems allowed for the generation of localized microstreaming which produced rapid mixing characteristics within a platform that is predominately laminar. The use of this phenomenon was integrated into a cell trapping and lysing system (Marmottant and Hilgenfeldt 2003) and thereafter harnessed for particle transport (Marmottant and Hilgenfeldt 2004; Marmottant et al. 2006). Particle transport was achieved through the use of asymmetrical flow caused by obstructions near the liquid-air interface of trapped air bubbles within etched surface cavities (Marmottant et al. 2006). The use of acoustic microstreaming was further studied to determine how interface oscillations are related to voltage and frequency input ( $\mathrm{Xu}$ and Attinger 2007). These observations were used to determine the optimal trapping geometry for a perpendicular-oriented cavity and that fast mixing could be achieved near the cavity interface ( $\mathrm{Xu}$ and Attinger 2007). In 2006, Dijkink et al. (2006) propelled an "acoustic scallop" within a liquid reservoir using a onesided sealed capillary tube and acoustic waves. The bubble's oscillations produced jet-like propulsion as fluid was expelled from the tube normal to the interface when the bubble expanded and pulled in symmetrically when it retracted. More recently, Ryu et al. reported on a technique for micropumping using an acoustically excited oscillating bubble for implantable microfluidic devices (Ryu et al. 2010).

We recently reported on the first proof-of-concept of a novel acoustic pumping platform that exploits trapped air bubbles within angled lateral cavities off a main microchannel to address the shortcomings of traditional pumping technologies (Tovar and Lee 2009). Acoustic energy is supplied to the system via a piezoelectric transducer (PZT) located beneath the microfluidic chip that can be powered through a battery or USB powered circuit. The acoustic energy actuates the angled, lateral cavities producing a rectified, first-order pulsatile flow to drive fluid within the main microchannel. The system is initially primed using capillary forces or a slight negative pressure at the outlet to draw fluid in and trap air bubbles within the lateral cavities. The main advantages of this pumping mechanism are its ability to drive fluid using only an external acoustic energy source and its easy integration with existing microfluidic devices. Since the main driving mechanism is the liquidair interface of the trapped air bubbles, fabrication is achieved through a one-step photolithography process with lateral cavities situated in the same plane as the microchannels. The fact that lateral cavity acoustic transducer (LCAT) pumps are dead-end side channels they can be implemented in a simple passive chip rendering it amenable to very low-cost manufacturing processes with potential for applications requiring disposable devices. In this article, we expand the acoustic pumping operational understanding and improve the pumping platform efficiency and operation through simulation and experimental results. We also implement a practical pumping system that is able to drive fluid from one chip to another demonstrating a real-world application.

\section{Acoustic pumping: principle and operation}

Acoustic microstreaming using air bubbles confined in a liquid medium uses sound waves to energize the gaseous phase to oscillate the liquid-air interface (Elder and Nyborg 1956). This streaming occurs due to first-order pulsatile flow from the oscillating bubble. The first-order pulsatile flow induces a second-order streaming flow due to the balance between viscous forces and the nonlinear inertial forces at the liquid-air bubble boundary (Elder and Nyborg 1956; Nyborg 1958; Lighthill 1978; Leighton 1997; Marmottant et al. 2006). The streaming velocity at the boundary layer can be described as:

$u_{\mathrm{s}} \sim \frac{U_{0}^{2}}{\omega R}$

where $u_{\mathrm{s}}$ is the steady-state flow velocity, $U_{0}$ is the oscillatory flow amplitude, $\omega$ is the angular frequency, and $R$ is the length along the boundary layer (Squires and Quake 2005; Marmottant et al. 2006).

Although streaming has been previously demonstrated to achieve rapid mixing (Liu et al. 2002), further applications have been limited due to the controllability of the streaming patterns. Previous work shows that controllability of the streaming was achieved by using lateral cavities (Tovar and Lee 2009) that acted as jet-like nozzles similar to an "acoustic scallop" (Dijkink et al. 2006). Using the first-order pulsatile flow generated by the oscillating liquid-air interface and angled lateral cavity pairs, rectified flow is achieved in a time-averaged manner. This valve-less micropumping phenomenon is achieved by means of the angled lateral cavities serving as a rectifying 
geometry to rectify flow. The flow rate of the micropump is a function of the PZT actuation amplitude and the number of cavity pairs. This working principle is only possible if the Reynolds number is large enough for inertia to be important (Dijkink et al. 2006). To estimate the Reynolds number near the angled lateral cavities, the peak Reynolds number of the oscillatory flow can be described as:

$R e=\frac{2 R_{\mathrm{c}} \omega A}{v}$,

where $A$ is the max liquid-air interface amplitude, $R_{\mathrm{c}}$ is the radius of the bubble in the cavity, $\omega$ is the angular frequency, and $v$ is the kinematic viscosity of the liquid being pumped (Dijkink et al. 2006). Using measured values, such as $R_{\mathrm{c}}=100 \mu \mathrm{m}, \omega=2 \pi \mathrm{f}, f=35 \mathrm{kHz}, A=5-10 \mu \mathrm{m}$, and $v=1 \times 10^{-6} \mathrm{~m}^{2} / \mathrm{s}$, calculated Reynolds number range of 220-440 is seen near the liquid-air interface. This operating principle shows that the pumping mechanism would begin failing as kinematic viscosity, $v$, approaches $2 \times 10^{-4} \mathrm{~m}^{2} / \mathrm{s}$.

\section{Design and fabrication}

\subsection{LCAT pump designs}

The LCAT pump design is based on a preliminary study showing proof-of-concept pumping within a microfluidic chip (Tovar and Lee 2009). The recirculation design is used here as a preliminary assessment of the individual characteristics of the paired lateral cavities within the microchannel. In the previous work, it was determined that the lateral cavities oriented at $15^{\circ}$ to the main microchannel performed at the best efficiency. The channel height is $100 \mu \mathrm{m}$ with a main microchannel width of $500 \mu \mathrm{m}$. The lateral cavities are $500-\mu \mathrm{m}$ long with widths of $100 \mu \mathrm{m}$ and spacing between each cavity at $100 \mu \mathrm{m}$. The dimensions of the device can be seen in Fig. 1a. The device length, excluding the inlet and outlet channels is $10.5 \mathrm{~mm}$ with a device width of $4.5 \mathrm{~mm}$. The three designs used to determine the pumping characteristics have 1,5 , and 10 cavity pairs on the main microfluidic channel and can be seen in Fig. 1a.

The behavior of the acoustic pumping mechanism using the design in Fig. 1a only demonstrates recirculatory pumping operation. To determine the pumping behavior with an open inlet and outlet, a scaled-up device is designed and shown in Fig. 1b. The 80 cavity pair serpentine design is fabricated with the same dimensions as the recirculation design (channel height of $100 \mu \mathrm{m}$, main microchannel width of $500 \mu \mathrm{m}$, lateral cavity lengths of $500 \mu \mathrm{m}$, widths of $100 \mu \mathrm{m}$, and a cavity to cavity spacing of $100 \mu \mathrm{m}$ ). The device footprint is $9 \times 12.3 \mathrm{~mm}$ with seven bends and eight straight sections with 10 cavity pairs per straight section. The inlet reservoir is fabricated using a $15 \mathrm{~mm}$ hole-punch and the outlet is fabricated using a $5 \mathrm{~mm}$ hole-punch. The total polydimethylsiloxane (PDMS) thickness is approximately $10 \mathrm{~mm}$. An image of the lateral cavities fabricated within the PDMS device is shown in Fig. 1c.
Fig. 1 a 1, 5, and 10 cavity pair designs with a channel width of $500 \mu \mathrm{m}$ and cavity widths of $100 \mu \mathrm{m}$ with cavity-to-cavity spacing of $100 \mu \mathrm{m}$. b The 80 cavity serpentine design is used to demonstrate non-

recirculation pumping. Fluid is introduced into the system at the inlet and is pumped out of the system at the outlet. The channel and cavity widths and cavity-to-cavity spacing are the same as the 1,5 , and 10 cavity pair design shown (a). c A microscope image of a LCAT pump with the main channel filled with DI water and polystyrene beads
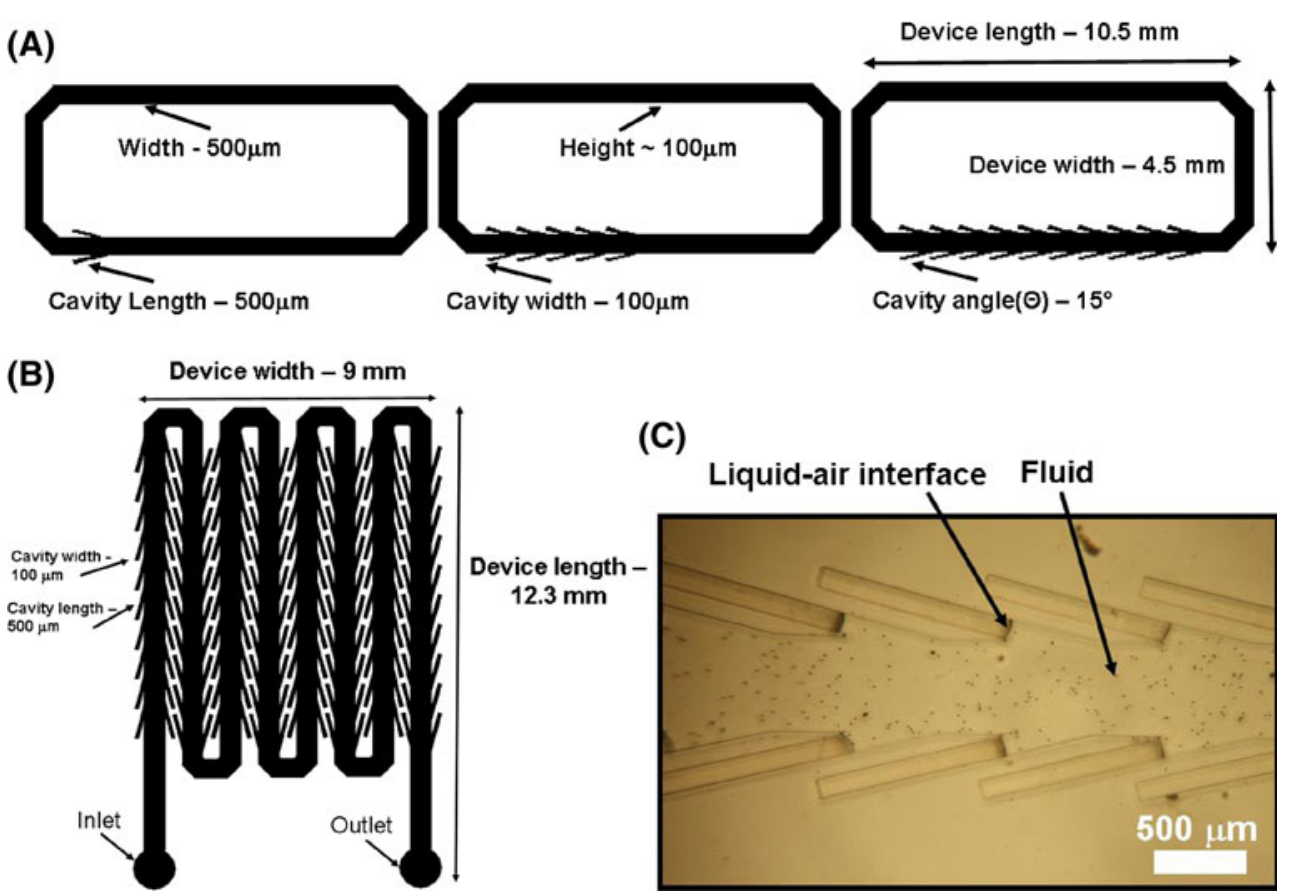

(C)

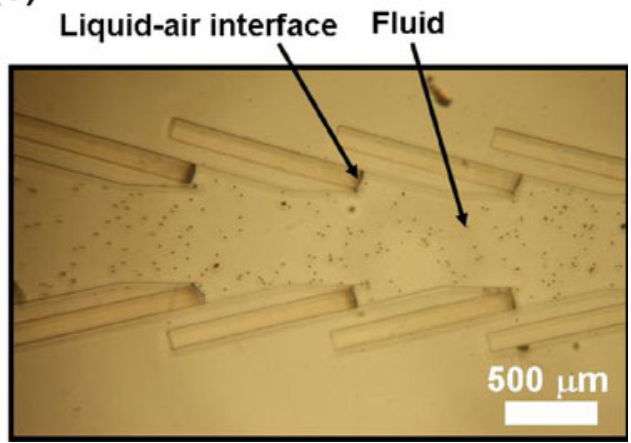




\subsection{Microfabrication of LCAT chip}

The microfluidic acoustic pumping devices are fabricated using standard soft lithography techniques (Duffy et al. 1998). The recirculation and 80 cavity serpentine designs are fabricated out of PDMS as the main polymer microchannel carrier with a glass microscope cover slip as the base substrate. The PDMS (Sylgard 184, Dow Corning Corp., USA) microchannels are molded from a SU-8 50 (Microchem Corp., USA) photoresist layer which is UV exposed and developed on a $3^{\prime \prime} \mathrm{Si}$ wafer to produce the desired microfluidic pattern. A standard 10:1 ratio of PDMS monomer to curing agent is used for all microdevices tested in these experiments. The PDMS is cured at $60^{\circ} \mathrm{C}$ for at least $4 \mathrm{~h}$ and are bonded to microscope coverslips $(150-\mu \mathrm{m}$ thick) after a 2 min plasma activation of the surfaces. The inlet and outlet holes are punched in the PDMS before bonding using a 5-mm diameter hole-punch. Upon bonding, the devices are kept stored for at least $24 \mathrm{~h}$ at room temperature before experimental operation to allow the PDMS surface to return to a hydrophobic state.

\section{Experimental setup}

\subsection{Operation of LCAT}

The LCAT devices are energized by placing them on a 15-mm diameter piezoelectric air transducer (SMUTF40TR15A, Stem Inc., USA) coated with an approximately $300 \mu \mathrm{m}$ thick layer of Aquasonic ${ }^{\circledR} 100$ ultrasound transmission gel (Parker Laboratories, Inc., USA) (Fig. 2a, b). The PZT is driven at $35 \mathrm{kHz}$ through a Krohn-Hite Model 7500 (Krohn-Hite Corp., USA) power amplifier with a frequency generation source from an Agilent 33220A (Agilent Technologies, Inc., USA) function generator. The PZT is fitted into a custom made acrylic housing so that the top surface of the PZT is flush with the acrylic surface as shown in Fig. 2a. The devices are placed on the surface and are activated for flow velocity measurements. The operational setup of the devices can be seen in Fig. 2 .

\subsection{Fluid flow velocity and pressure measurements}

The characterization of the acoustic pumping devices is done through measuring the fluid flow velocity of the recirculation designs as the voltage amplitude applied to the PZT is increased. The fluid flow velocity is measured through the use of 2-6 $\mu \mathrm{m}$ polystyrene, non-fluorescent microbeads (F13838, Invitrogen, USA). The polystyrene beads are mixed with DI water with a final density of $3 \times 10^{5}$ beads $/ \mathrm{ml}$ and are drawn into the recirculation design through the inlet while applying a slight negative pressure at the outlet. As the fluid is drawn into the channels, visual inspection is done to confirm that the channels are filled with the microbead/DI water mixture. The device is placed on the custom made PZT platform using a thin layer of ultrasound gel and is visualized through a Nikon Eclipse L150 upright microscope. A high speed camera is used to capture the fluid flow within the device upon activation of the PZT. The high speed cameras used in the experiments are a FASTCAM PCI (Photron USA, USA) that is capable of video capture up to $10,000 \mathrm{fps}$ and a Phantom 7.3 (Vision Research, Inc., USA) capable of video capture up to 500,000 fps. Fluid flow velocity is measured through the tracking of multiple microbeads within the fluid flow over a specified period of time. The distance of each bead is measured through the stacking of images and the use of Image $\mathrm{J}$, an image analysis program by $\mathrm{NIH}$ Image.

The pressure generation by the 80 cavity serpentine device is calculated through measuring the height difference of the liquid columns between the inlet and the outlet (Wang et al. 2009). A large reservoir is used as the inlet and the outlet is connected to tygon tubing extending perpendicular to the surface of the device. The device is primed with DI water and allowed to equalize so there is no residual flow before operation. The activation of the PZT

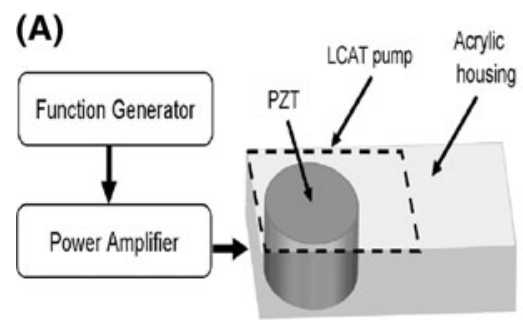

Fig. 2 a The PZT is housed within an acrylic housing and is operated through an Agilent 33220A function generator with a Krohn-Hite 7500 power amplifier driving the PZT. The black dotted line represents where the LCAT device is placed on the acrylic surface.

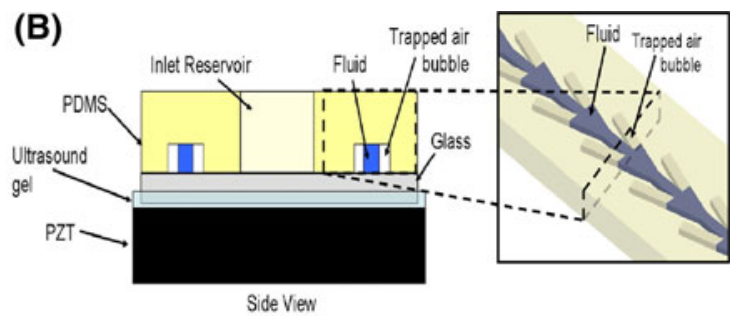

b The PDMS device is bonded onto a glass microscope coverslip, which is placed on the top of a PZT coated with ultrasound gel. The black dotted line in the 3D rendition shows a partial slice of where the side view is visualized 
driven acoustic pump drives the fluid up the tygon tubing until a maximum pressure head is reached. The PZT is activated at specified voltages for $30 \mathrm{~min}$ to allow the maximum height to be reached. A Rebel XSi DSLR with a $100 \mathrm{~mm} \mathrm{f} / 2.8$ macro lens (Canon Inc., Japan) is used to record images throughout the experiment. The height difference is measured using Image $\mathbf{J}$ and a known dimension in the image for scale reference. The experimental setup can be seen in the Supplementary material 1 .

\subsection{Simulations}

The fluid flow characteristics of the acoustic pumping platform are investigated using computational fluid dynamics with CFD-ACE+ v2008 (ESI Group, Inc., France). The LCAT model is setup using the fluid flow, grid deformation, volume of fluid (VOF), and particle spray modules using a structured mesh throughout. The fluid in the model is treated as an incompressible Newtonian fluid and no mechanical properties were given to the model walls. Figure 3 shows the 3D model used for the simulation of the device with two open outlets. The device is modeled with a single cavity and uses a symmetrical boundary to mirror fluid characteristics at the symmetry plane which simulates a single cavity pair LCAT pumping device and consists of 20,680 cells. The designed cavity length is $500 \mu \mathrm{m}$ and the width is $100 \mu \mathrm{m}$ and the microchannel height is $100 \mu \mathrm{m}$. A no-slip boundary condition is applied to all walls in the model which is every surface besides the symmetrical boundary and the two outlet interfaces. The fluids used in the model are air and water with density $(\rho)$ and viscosity $(\mu)$ values of 1.1614 and $997 \mathrm{~kg} / \mathrm{m}^{3}$ and $1.846 \times 10^{-5}$ and $0.855 \times 10^{-3} \mathrm{~Pa}$ s. The region labeled " 1 " and outlined in red in Fig. 3 is initially air while the region labeled " 2 " and outlined in blue is initially water.
The contact angle is set with respect to water and values are used for both PDMS (top and side surfaces) and glass (bottom surface). The bottom surface, glass, has an $80^{\circ}$ contact angle with water while the top and side surfaces, PDMS, are at a $95^{\circ}$ contact angle with water. A surface tension, $\sigma$, of $72 \times 10^{-3} \mathrm{~N} / \mathrm{m}$ is used for the water in contact with air in the model. To simulate an oscillating pressure within the chamber of the cavity from an acoustic energy source, a moving wall is placed in the back of the lateral cavity as shown in Fig. 3. The wall is oscillated at an amplitude $A$ of $500 \mathrm{~nm}$ and is given the following function, $y(t)=A \sin (2 \pi f t)$, where $y(t)$ is the displacement in $y$ direction with respect to time, the oscillation frequency $f=35 \mathrm{kHz}$, and $t$ is the time in seconds. The wall amplitude is chosen from experimental observations of the liquid-air interface oscillations at $25 \mathrm{~V}_{\mathrm{pp}}$. Since CFDACE + will not solve for the resonance frequency of the trapped air bubble, a high speed camera is used to capture the liquid-air interface oscillation and is compared to varying wall displacements. The final amplitude of $500 \mathrm{~nm}$ is chosen for the wall amplitude as this displacement produces liquid-air interface oscillations in the simulation that are comparable to those observed during experiments (Supplementary material 2). A transient solver is used with an automatic time step with a minimum and maximum time step of 0 and $2 \times 10^{-6} \mathrm{~s}$ with Euler time accuracy and an initial time step of $1 \times 10^{-9} \mathrm{~s}$. Gravity, rotation, and chimera grid are left off and the reference pressure and temperature are set at $100,000 \mathrm{~N} / \mathrm{m}^{2}$ and $300 \mathrm{~K}$. Drag is included in the spray module with drag coefficient set to incompressible. The inlets are treated as walls with bounce normal CR and parallel CR set to 1. All fluid initial velocities are set to $0 \mathrm{~m} / \mathrm{s}$. The solver conditions are set to a max of 50 iterations with a convergence criteria of 0.0001 and a minimum residual of $1 \times 10^{-18}$. Spatial differencing
Fig. 3 Single pair LCAT design and setup for CFDACE + simulation. The red region labeled " 1 " represents the initial conditions of air, while the blue region labeled "2" is initially DI water. A single cavity is placed in the model with a symmetry boundary to simulate an opposing cavity in operation. A structured grid is used for the entire design with grid deformation to simulate the oscillating pressure from an external PZT. (Color figure online)

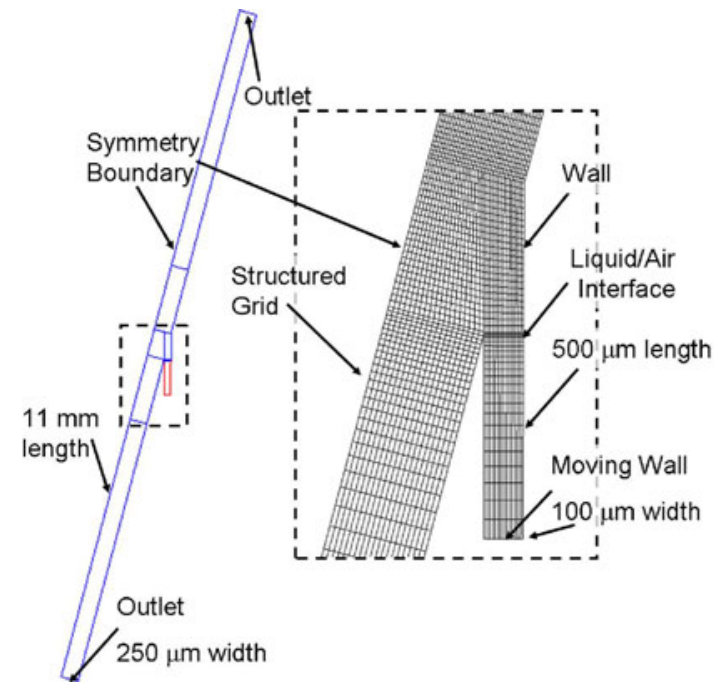


for velocity is set to upwind and inertial relaxation for velocities is set to 0.2 and linear relaxation for pressure, density, and viscosity is set to 1 . The total time run for the simulation is set to $t=3 \mathrm{~ms}$ with a total elapsed CPU time of $2,255 \mathrm{~min}$ to finish the simulation. This allows for approximately 105 cycles to occur at the liquid-air interface. To track the fluid flow within the device, $2-4 \mu \mathrm{m}$ polystyrene beads are placed throughout the model.

\section{Results and discussion}

\subsection{Pumping velocities and pressure output}

The measured pumping pressure generated by the 1,5 , and 10 lateral cavity pair recirculation devices is shown in Fig. 4. The figure shows the pressure generated at $0,5,10,15,20$, and $25 \mathrm{~V}_{\mathrm{pp}}$ applied to the PZT at an optimal pumping frequency of $35 \mathrm{kHz}$. As the voltage amplitude applied to the PZT is increased, the pumping velocity and pressure generated increases quadratically. This quadratic relationship has been observed in previous literature ( $\mathrm{Xu}$ and Attinger 2007) and corresponds to the second-order streaming flow consistent with acoustic microstreaming. To determine the pressure generated by the cavity pairs, the hydrodynamic resistance is calculated for the recirculation design and is used in conjunction with Poiseuille's law, $P=Q \times R$. The resistance of the design is calculated using the hydrodynamic resistance equation for a rectangular, cross-sectional channel and is compared to a CFD analysis of the design for confirmation. The equation used to determine the resistance is

$R_{\mathrm{H}}=\frac{12 \mu L}{w h^{3}\left(1-0.630 \frac{h}{w}\right)}$,

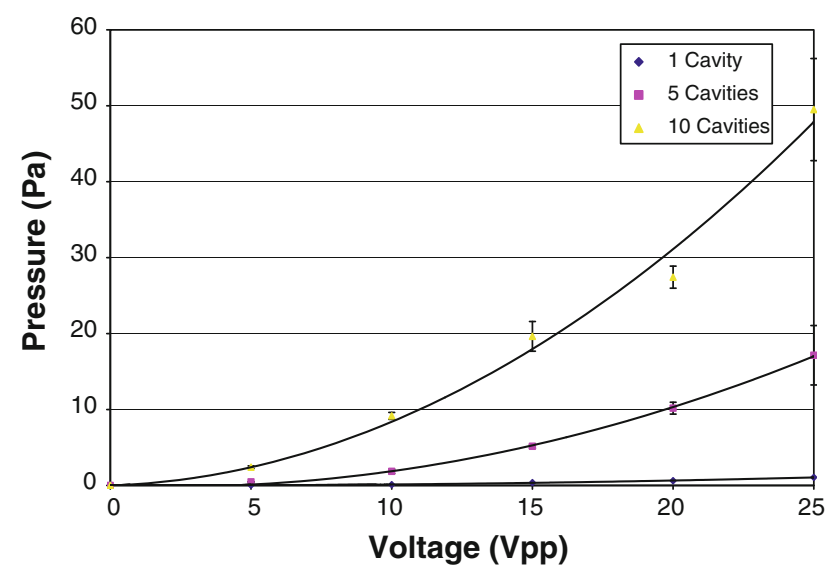

Fig. 4 The pumping pressure generated by a 1, 5, and 10 cavity pair device with a maximum input voltage of $25 \mathrm{~V}_{\mathrm{pp}}$. The pressure output is calculated using the measured average flow velocity and the calculated hydrodynamic resistance of the microfluidic system where $\mu$ is the viscosity, $L$ is the channel length, $w$ is the channel width, and $h$ is the channel height (Lemoff and Lee 2000). The calculated resistance for the system is found to be approximately $6.5 \times 10^{11} \mathrm{~Pa} \mathrm{~s} \mathrm{~m}^{-3}$. The maximum pumping velocity of the 10 cavity pair device is $1.6 \mathrm{~mm} \mathrm{~s}^{-1}$ at a PZT voltage of $25 \mathrm{~V}_{\mathrm{pp}}$, which correlates to a flow rate of $4.6 \mu \mathrm{min}^{-1}$ with a calculated pressure generation of $49 \mathrm{~Pa}$. The maximum pumping velocity for a single cavity pair is $35 \mu \mathrm{m} \mathrm{s}^{-1}$ with a calculated pressure generation of $1 \mathrm{~Pa}$ at $25 \mathrm{~V}_{\mathrm{pp}}$.

As reported in previous work (Tovar and Lee 2009), while the voltage amplitude is increased, the interface oscillations become too large to maintain their fixed position in the lateral cavity. This causes instability in the pumping profile and ultimately leads to device failure as the trapped bubbles expand out into the main microchannel. Defining the voltage amplitude limits allows a further understanding of the LCAT pumping predictability. Using the pumping pressure generation data, a set of equations are developed to predict the pressure generation for designs with more than 10 cavity pairs and are shown in Fig. 5. These equations are derived from correlating the pressure generation to the number of cavity pairs for each voltage input. The equations for each voltage input are used to predict the pumping performance of an 80 cavity pair serpentine design. In the original work done on particle transport and acoustic pumping (Marmottant et al. 2006; Tovar and Lee 2009), the performance is investigated in an enclosed cuvette and in a microfluidic recirculatory design, respectively. These works demonstrated recirculatory pumping and particle transport, however, did not confirm the ability to perform dead volume pumping. The 80 cavity

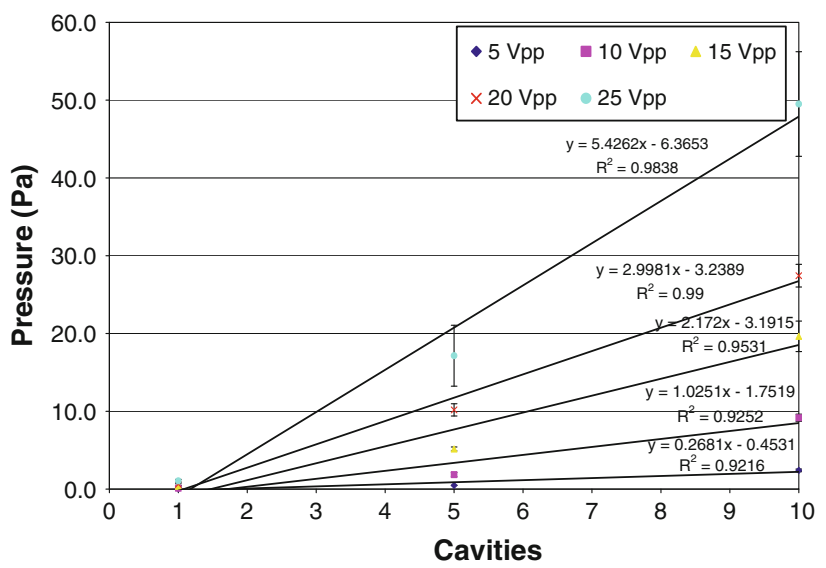

Fig. 5 The graph shows the correlation between the number of cavity pairs and the voltage applied to the system in term of pressure generation. The fitted equations are used to determine the estimated pressure output for a scaled-up platform such as that in the 80 cavity serpentine design 
pair design is used to confirm that rectified flow is achieved through the angled lateral cavity pairs.

Using the 80 cavity pair serpentine design, maximum pressure generation is calculated using a height difference between two fluid columns. The pressure generated through the pumping is plotted in blue $(\diamond)$ in Fig. 6 . The maximum pressure generated is $350 \mathrm{~Pa}$ with a voltage of $25 \mathrm{~V}_{\mathrm{pp}}$. The predicted pressure generation is compared to the experimental data in the same figure and is plotted in red (ם). As shown in the figure, the predicted and experimental data closely match except for a variation at $20 \mathrm{~V}_{\mathrm{pp}}$ and $25 \mathrm{~V}_{\mathrm{pp}}$. This is thought to be due to the long operation of the device, typically greater than $30 \mathrm{~min}$, as the voltage is ramped up from 0 to $25 \mathrm{~V}_{\mathrm{pp}}$. Prolonged operation of the device causes a decrease in performance as the trapped air bubbles expand from their cavities into the main microchannel. If the pump is not being operated, the trapped air in the cavity diffuses through the PDMS. As the bubbles expand into the microchannel or diffuse through the PDMS, the performance decreases since the oscillating interface is no longer perpendicular to the lateral cavity or the distance is farther from the cavity opening. If the bubble completely expands into the main microchannel, the pump will no longer operate due to the occluded channel.

\subsection{Acoustic pumping flow paths}

The fluid streamlines produced during the operation of a single cavity pair device are shown in Fig. 7a, b. The streamlines are visualized from the CFD-ACE+ transient solution using the parameters described in Sect. 4.3. Figure 7 a shows the main flow streamlines generated as the interface oscillations commence. Fluid flow is either pushed away or pulled toward the interface as the liquidair interface oscillates. The recirculation zone near the

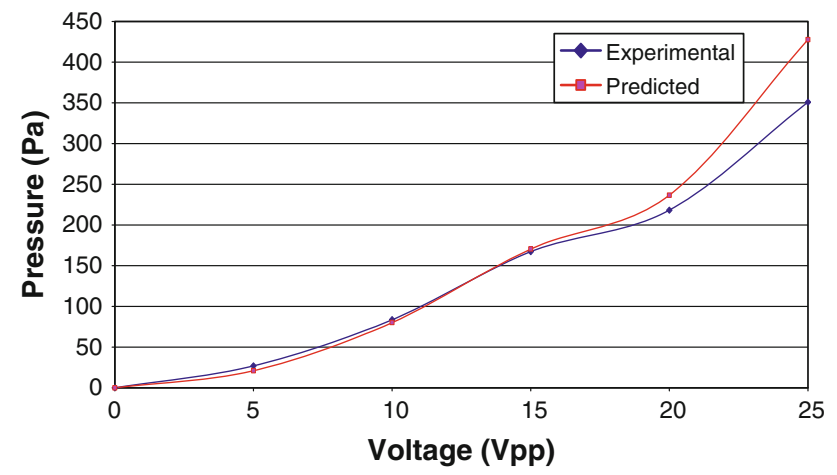

Fig. 6 The pressure generated by an 80 cavity serpentine design measured through a liquid height differential of DI water in tygon tubing. The observed pressure output $(\bullet)$ closely matches the calculated pressure output $(\boldsymbol{\square})$ as extrapolated from the fitted equations shown in Fig. 5 for this 80 cavity serpentine design liquid-air interface (Fig. 7b) is the time-averaged flow produced by the nonlinear inertial forces (Lighthill 1978; Leighton 1997; Marmottant et al. 2006). Coupled with the recirculation flow, the time-averaged bulk flow is confirmed with particle tracing within the system and can be seen in Fig. 8. An observation made in both simulation and experimental results is the high flow velocity of fluid and particles near the liquid-air interface and can be seen in the video (Supplementary material 3 ). The recirculation streaming near the interface is consistent with traditional acoustic microstreaming work (Liu et al. 2002; Marmottant and Hilgenfeldt 2004; Marmottant et al. 2006; Xu and Attinger 2007), however, fluid flow away from the cavity interface confirms rectified flow from the angled lateral cavity design.

Figure 8 shows a time lapsed image of a simulated acoustic pumping device with $4 \mu \mathrm{m}$ polystyrene beads spread throughout the main channel. The paths of the beads are visualized using the particle trajectory history tool in CFD-VIEW. As seen in the figure, the beads located near the liquid-air interface travel a much greater distance than the particles fore and aft of the cavity interface. The average distance travelled for each particle in the upstream and downstream channel is approximately $1 \mu \mathrm{m}$ over $8.29 \mathrm{~ms}$. This gives an average particle velocity of approximately $120 \mu \mathrm{m} / \mathrm{s}$ for a single cavity pair. When comparing this to experimental data, it can be seen that the simulated velocity is approximately 3.5 times larger. However, the current 3D model is only $11 \mathrm{~mm}$ in length, while the experimental design is approximately $30 \mathrm{~mm}$ in length in a recirculation system. Since for a given flow rate, pressure is directly proportional to fluidic resistance and the resistance is proportional to channel length, it can be estimated that the flow velocity is approximately three times smaller than the simulated value. The corrected velocity would be in good agreement with the experimental data.

\subsection{Chip-to-chip pumping}

Expanding on the observations made through both experimental and simulation studies, the 80 cavity pair serpentine design is used to drive fluid from one chip to another. This pumping demonstration shows positive pressure generation from an acoustic microstreaming operated device. The rectified flow allows for fluid to be pumped from the LCAT pumping region out through a $1 \mathrm{~mm}$ ID tygon tube connected to an external straight channel microfluidic chip. The chip is operated in the same manner as described in Sect. 4 with the DI water dyed red for visualization purposes. The video can be seen in the Supplementary material 4 and shows the pump being actively turned off and on various times throughout its operation. 


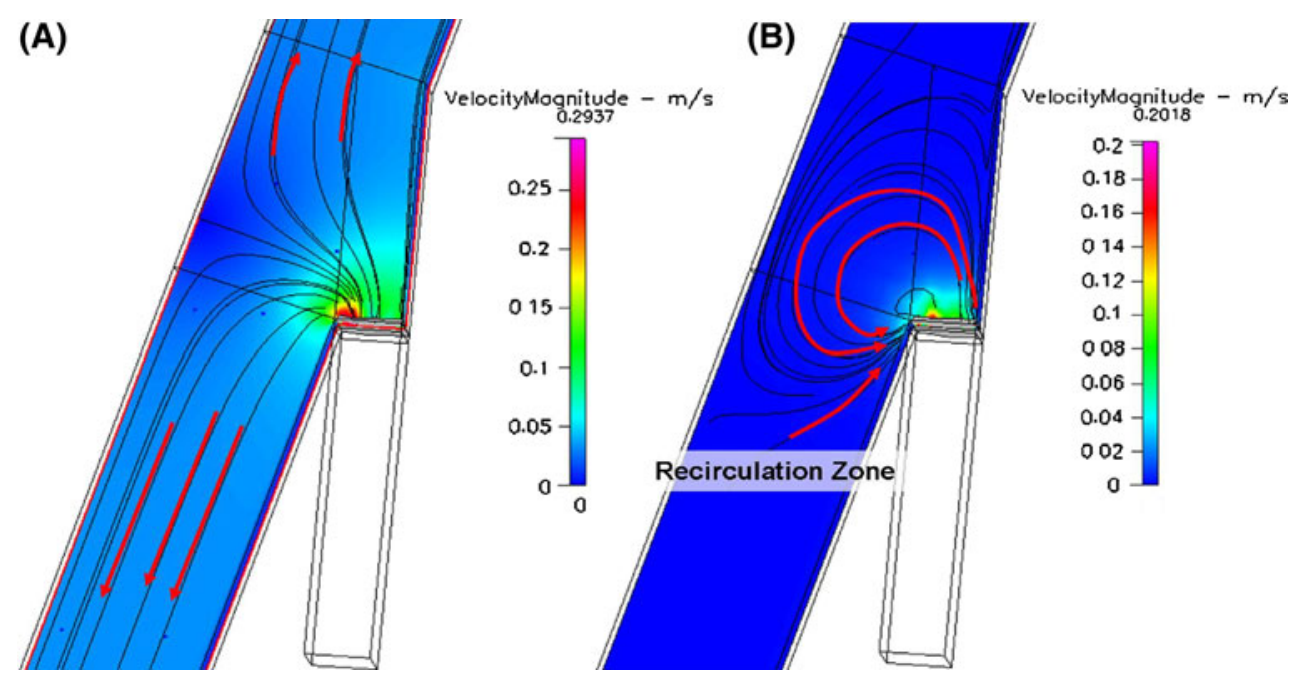

Fig. 7 Instantaneous flow velocity magnitude coupled with fluid streamlines for a single-lateral cavity pair device. The fluid streamlines show the two main streaming patterns seen within the system. a As the interface begins to oscillate, the instantaneous flow velocities at the interface is at its maximum velocity and fluid flow paths flow away from the interface region while $\mathbf{b}$ recirculation is produced near the liquid-air interface as the interface retracts. Over a transient solution, the oscillating membrane draws fluid into the lateral cavity region and expels it out perpendicular to the liquid-air interface and downstream
Fig. 8 The CFD-ACE model is used for confirmation of the general fluid flow path by tracking $4 \mu \mathrm{m}$ polystyrene particles within the microchannel as the cavity pressure is oscillated as described in Sect. 4.3. A transient solution is solved for approximately $8.29 \mathrm{~ms}$ with the particles traveling a distance of $1 \mu \mathrm{m}$. The higher bead velocity near the liquid-air interface is representative of traditional acoustic microstreaming, however, bead movement away from the interface demonstrates rectified flow. These simulation results closely match the experimental observations seen in Supplementary material 3

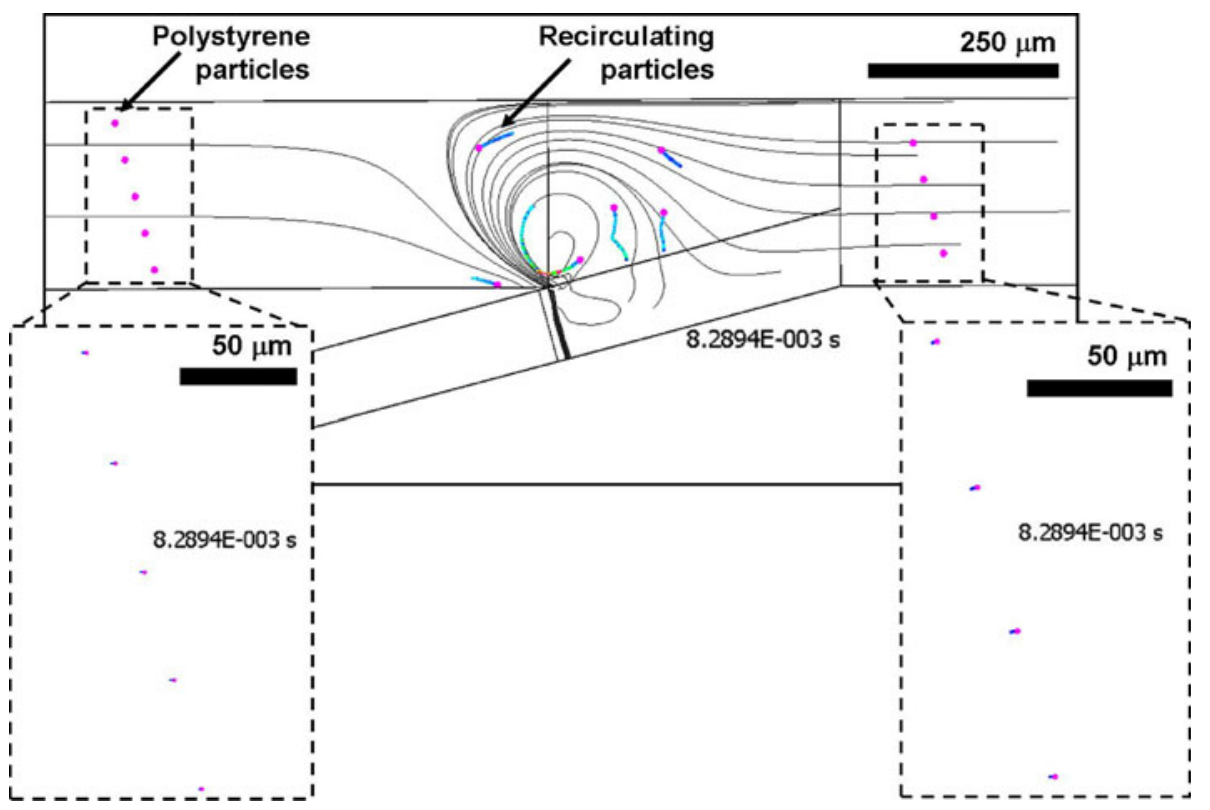

\section{Future works and improvements}

This report has demonstrated and investigated the use of trapped air bubbles and acoustic energy as an effective fluidic driving mechanism. The simplistic design and ease of operation lends itself to a variety of microfluidic applications that would need an integrated and portable pumping source. One major hindrance in the development of microfluidic pumps is the ability to demonstrate its operation in a portable system while still maintaining simple fabrication. The demonstration of portability was done by developing a prototype acoustic driving platform that is powered by 4 AA batteries or a USB 2.0 port. Two 80 cavity pumps are fabricated and bonded to a glass coverslip which is placed on the PZT. Each pump has a reservoir of approximately $100 \mu$, which are filled with either a red or green dye solution. The fluid is initially drawn in with capillary forces or a slight negative pressure at the outlet for priming and the pumps are then activated using the PZT. The square-wave driving frequency is $35 \mathrm{kHz}$ at an amplitude of $25 \mathrm{~V}_{\mathrm{pp}}$. As seen in the video (Supplementary material 5), each pump drives fluid to the outlet of the 
system and can be independently controlled. This prototype device demonstrates the use of the acoustic pumping platform and its extendibility into a portable system for serial delivery of reagents.

\section{Conclusions}

This article demonstrates a novel acoustically activated microfluidic pumping platform that is both simple to fabricate and operate. The pumping is generated through rectified flow from oscillating liquid-air interfaces within lateral cavities oriented $15^{\circ}$ to the main microchannel. The device is fabricated using only a single lithography step and adds no additional fabrication processes when integrating into existing microfluidic systems. Lateral cavities of dimensions $100 \mu \mathrm{m}$ wide by $500 \mu \mathrm{m}$ long and a height of $100 \mu \mathrm{m}$ are activated at a pumping frequency of $35 \mathrm{kHz}$ and PZT voltages ranging from 0 to $25 \mathrm{~V}_{\mathrm{pp}}$. The pumping platform is characterized through determining a relationship between cavity pairs and voltage input and is predicted beyond 10 pairs to 80 cavity pairs. The maximum pumping pressure generated with the 80 cavity pair device is approximately $350 \mathrm{~Pa}$ at $25 \mathrm{~V}_{\mathrm{pp}}$. Simulation work is also done to gain a better understanding of the fluid behavior near the liquid-air interface and to determine if the model can be used as an aid in future designs and iterations. The liquid-air interface oscillations in the CFD model are matched through experimental observations and fluid flow velocity is found to be in good agreement to a single cavity pair design. To further demonstrate the practicality of the LCAT pumping mechanism, a two fluid pumping device is fabricated and used to show multi-fluid pumping from large reservoirs at the inlets to a single channel outlet. To extend the applications that could be made possible through the use of the LCAT platform, a portable acoustic generation device is designed that can be operated on either 4 AA batteries or a standard USB 2.0 port.

Acknowledgments The authors acknowledge financial support from the DARPA S\&T Program (HR0011-06-1-0050) through the Micro/nano Fluidics Fundamentals Focus (MF3) Center.

Open Access This article is distributed under the terms of the Creative Commons Attribution Noncommercial License which permits any noncommercial use, distribution, and reproduction in any medium, provided the original author(s) and source are credited.

\section{References}

Ahmed D, Mao X, Juluri B et al (2009a) A fast microfluidic mixer based on acoustically driven sidewall-trapped microbubbles. Microfluid Nanofluid 7(5):727-731

Ahmed D, Mao X, Shi J et al (2009b) A millisecond micromixer via single-bubble-based acoustic streaming. Lab Chip 9(18):2738-2741
Chang ST, Beaumont E, Petsev DN et al (2008) Remotely powered distributed microfluidic pumps and mixers based on miniature diodes. Lab Chip 8(1):117-124

Chiu SH, Liu CH (2009) An air-bubble-actuated micropump for onchip blood transportation. Lab Chip 9(11):1524-1533

Chung SK, Cho SK (2008) On-chip manipulation of objects using mobile oscillating bubbles. J Micromech Microeng 18(12): 125024

Chung S, Cho S (2009) 3-D manipulation of millimeter-and microsized objects using an acoustically excited oscillating bubble. Microfluid Nanofluid 6(2):261-265

Dijkink R, Van der Dennen J, Ohl CD et al (2006) The acoustic scallop': a bubble-powered actuator. J Micromech Microeng 16: $1653-1659$

Duffy DC, McDonald JC, Schueller OJA et al (1998) Rapid prototyping of microfluidic systems in poly(dimethylsiloxane). Anal Chem 70(23):4974-4984

Elder S, Nyborg WL (1956) Acoustic streaming resulting from a resonant bubble. J Acoust Soc Am 28(1):155

Gervais L, Delamarche E (2009) Toward one-step point-of-care immunodiagnostics using capillary-driven microfluidics and PDMS substrates. Lab Chip 9(23):3330-3337

Hettiarachchi K, Talu E, Longo ML et al (2007) On-chip generation of microbubbles as a practical technology for manufacturing contrast agents for ultrasonic imaging. Lab Chip 7(4):463-468

Kao J, Wang X, Warren J et al (2007) A bubble-powered micro-rotor: conception, manufacturing, assembly and characterization. J Micromech Microeng 17:2454-2460

Laser DJ, Santiago JG (2004). A review of micropumps. J Micromech Microeng (6):R35

Leighton T (1997) The acoustic bubble. Academic Press, San Diego

Lemoff AV, Lee AP (2000) An AC magnetohydrodynamic micropump. Sens Actuators B 63:178-185

Lighthill J (1978) Acoustic streaming. J Sound Vib 61(3):391-418

Liu RH, Yang J, Pindera MZ et al (2002) Bubble-induced acoustic micromixing. Lab Chip 2(3):151-157

Liu R, Lenigk R, Grodzinski P (2003a) Acoustic micromixer for enhancement of DNA biochip systems. J Microlithogr Microfabr Microsyst 2:178

Liu RH, Lenigk R, Druyor-Sanchez RL et al (2003b) Hybridization enhancement using cavitation microstreaming. Anal Chem 75(8):1911-1917

Marmottant P, Hilgenfeldt S (2003) Controlled vesicle deformation and lysis by single oscillating bubbles. Nature 423(6936): $153-156$

Marmottant P, Hilgenfeldt S (2004) A bubble-driven microfluidic transport element for bioengineering. Proc Natl Acad Sci USA 101(26):9523-9527

Marmottant P, Raven JP, Gardeniers H et al (2006) Microfluidics with ultrasound-driven bubbles. J Fluid Mech 568:109-118

Nyborg WL (1958) Acoustic streaming near a boundary. J Acoust Soc Am 30(4):329-339

Rife JC, Bell MI, Horwitz JS et al (2000) Miniature valveless ultrasonic pumps and mixers. Sens Actuators A 86(1-2):135-140

Ryu K, Chung SK, Cho SK (2010) Micropumping by an acoustically excited oscillating bubble for automated implantable microfluidic devices. J Assoc Lab Autom 15(3):163-171

Squires T, Quake S (2005) Microfluidics: fluid physics at the nanoliter scale. Rev Mod Phys 77(3):977-1026

Tho P, Manasseh R, Ooi A (2007) Cavitation microstreaming patterns in single and multiple bubble systems. J Fluid Mech 576: $191-233$

Tovar AR, Lee AP (2009) Lateral cavity acoustic transducer. Lab Chip 9(1):41-43

Tsai J, Lin L (2002) A thermal-bubble-actuated micronozzle-diffuser pump. J Microelectromech Syst 11(6):665-671 
Unger MA, Chou HP, Thorsen T et al (2000) Monolithic microfabricated valves and pumps by multilayer soft lithography. Science 288(5463):113-116

Wang S, Huang X, Yang C (2009) Valveless micropump with acoustically featured pumping chamber. Microfluid Nanofluid 8(4):549-555

Xu J, Attinger D (2007) Control and ultrasonic actuation of a gasliquid interface in a microfluidic chip. J Micromech Microeng 17(3):609-616
Zeng SL, Chen CH, Mikkelsen JC et al (2001) Fabrication and characterization of electroosmotic micropumps. Sens Actuators B 79(5):107-114

Zimmermann M, Hunziker P, Delamarche E (2009) Autonomous capillary system for one-step immunoassays. Biomed Microdevices $11(1): 1-8$ 